\title{
Isospectral shapes with Neumann and alternating boundary conditions
}

\author{
T.A. Driscoll ${ }^{1, *}$ and H.P.W. Gottlieb ${ }^{2, * *}$ \\ ${ }^{1}$ Department of Mathematical Sciences, University of Delaware, Newark, DE 19716 \\ ${ }^{2}$ School of Science, Griffith University, Nathan, Queensland 4111, Australia
}

(May 2003)

The isospectrality of a well-known pair of shapes constructed from two arrangements of seven congruent right isosceles triangles with the Neumann boundary condition is verified numerically to high precision. Equally strong numerical evidence for isospectrality is presented for the eigenvalues of this standard pair in new boundary configurations with alternating Dirichlet and Neumann boundary conditions along successive edges. Good agreement with theory is obtained for the corresponding spectral staircase functions. Strong numerical evidence is also presented for isospectrality in an example of a different pair of shapes whose basic building-block triangle is not isosceles. Some possible confirmatory experiments involving fluids are suggested.

DOI:

PACS numbers: 03.65.Ge, 02.70.-c, 46.70, 05.45.-a 


\section{INTRODUCTION}

As the most accessible realization of the negative answer to Kac's [1] question "Can one hear the shape of a drum?", the pair of isospectral shapes discovered by Gordon et al. [2] (termed respectively "bilby" and "hawk" in Figure 1), have subsequently been investigated from a variety of mathematical, numerical, and experimental viewpoints. Chapman [3] showed how domain eigenfunctions can be mapped from the constituent triangles of one shape to the second to prove isospectrality by transplantation, and described a proof by paper-folding. More recently, Okada and Shudo [4] have investigated isospectrality through a technique of successive unfolding of fundamental building block shapes and transplantation of eigenfunctions. Wu et al. [5] achieved a proof by an explicit mode matching method.

The numerical problem concerning the eigenvalues corresponds to solving an eigenvalue problem for the two-dimensional Helmholtz equation subject to the Dirichlet boundary condition (Dbc). Wu et al. [5] verified isospectrality numerically by an extrapolated mode-matching method, apparently to about 8 significant figures, tabulated for the first 25 modes. The "analytical" 9th and 21st modes there, corresponding to known simple modes of the underlying triangles, were not computed but were taken at their exact values. Subsequently, Driscoll [6], using a much more accurate modified domain-decomposition method, verified isospectrality numerically to 12 significant figures for the first 25 modes, including the two "analytical" modes for which the computation was more or less exact. This work showed that the computed results in Ref. [5] were actually accurate to about 4-5 significant figures.

On the experimental side, Sridhar and Kudrolli [7] performed measurements 
on thin microwave cavities of the appropriate shapes, utilizing the correspondence with a 2-dimensional Helmholtz equation in the electromagnetic formulation. Then Even and Pieranski [8] constructed actual shaped small "drums" - membranes made from liquid crystal smectic films - and measured their vibrations.

In this paper, we investigate numerical aspects of the isospectrality of the two standard bilby and hawk shapes, as well as other shapes, when Neumann boundary conditions (Nbc) are present, and make suggestions for possible experimental verification.

\section{NEUMANN BOUNDARY CONDITIONS}

The most commonly encountered boundary condition is the Dirichlet bc $\psi=0$ on the boundary. This corresponds to the standard "drum" condition for a vibrating membrane with fixed edges, as well as to the boundary condition for quantum billiards [9]. The Neumann bc $\partial \psi / \partial \mathrm{n}=0$ also has important manifestations [9], especially in acoustics where the pressure satisfies the Helmholtz equation with Nbc at a rigid boundary and for water surface waves. In electromagnetism also, the magnetic field of the transverse electric (TE) mode in a cavity has Nbc. The $\mathrm{Nbc}$ corresponds to the vibrational modes of a drum with stress-free edges, as discussed by Hobiki et al. [10], who numerically investigated such a situation for fractal boundary shapes. Russ et al. [11] also considered fractal resonators with Nbc numerically, remarking that this situation could represent transverse acoustical phonons of a $2 \mathrm{D}$ irregular crystallite. In the field of quantum billiards, Gremaud and Jain [12] considered rational and irrational rhombus billiards with Nbc. Kohler and Blumel [13] considered ray-splitting billiards including Nbc. Wiersig [14] has used the fact that, 
for barrier billiards, the Nbc pertains on part of the boundary of a symmetry-reduced polygon for the even states of the full shape. Neumann boundary conditions are therefore important, and the problem of isospectral shapes with these conditions will now be addressed.

The spectra under consideration in this section are the eigenvalues obtained from the two-dimensional Helmholtz equation $\left(\nabla^{2}+\mathrm{E}\right) \psi=0$ together with the $\mathrm{Nbc}$ on the edges. In an earlier proof of the existence of isospectral domains in dimensions greater than or equal to 4, Urakawa [15] already showed isospectrality for Dirichlet and for Neumann boundary conditions. In their announcement of Dbc isospectral twodimensional domains, Gordon et al [16] also stated that the same shapes with Nbc were isospectral; Okada and Shudo [4] noted that their procedures, suitably modified, prove isospectrality for Nbc. Chapman [3] proved isospectrality for the bilby/hawk and other pairs having the Nbc.

Gottlieb and McManus [17] produced explicit eigenfunctions and corresponding exact eigenvalues for some Nbc modes of the two isospectral shapes. From the nodal patterns of these analytical expressions and numerical solutions for low modes, they were able to identify the first five analytical Neumann modes as the 5th, 9th, 15th, 20th and 29th nontrivial sequential modes. The finite element computations mentioned there, whilst sufficient for identifying patterns, only verified isospectrality to about three significant figures. The exact modes mentioned above, however, serve as benchmarks in any investigation of $\mathrm{Nbc}$ isospectrality for these shapes.

A numerical verification of isospectrality of the two standard shapes of Figure 1 for Nbc, such as has been done previously for Dbc [5], [6] does not seem to have been carried out before. For this paper, the earlier work of Driscoll [6] has been 
adapted to the Nbc case. In [6] a candidate eigenfunction near a corner with interior angle $\pi / \alpha$ is expanded in local polar coordinates as $\sum_{n=1}^{M} c_{n} J_{n \alpha}(r \sqrt{E}) \sin (n \alpha \theta)$. Then one finds an eigenvalue $E$ by matching different expansions along the interfaces of a domain decomposition; numerically, this becomes minimization of the result of a matrix eigenvalue problem. For $\mathrm{Nbc}$ we replace the sine by a cosine and start the summation at $n=0$.

We have verified isospectrality in the Nbc configuration for the first 30 (nonzero) modes to 12 significant figures. The results for both shapes are given in Table I. With fundamental length unit 2 , the analytical modes described above have eigenvalues given by $E_{m, n}=\left(\pi^{2} / 4\right)\left(m^{2}+n^{2}\right) ; m \leq n=0,1,2, \ldots$ The cases $(m, n)=(0,1)$, $(1,1),(0,2),(1,2),(2,2)$ corresponding to the five analytical modes described above, together with their readily identifiable nodal patterns [17], are essentially recovered exactly. The nodal patterns of the 4th nontrivial mode (which is non-analytical) are shown in Figure 2.

It may be noted that many of the investigations concerned with quantum chaotic spectral statistics [9] deal with very large numbers of very high levels. The accuracy on these typically was $10^{-2}$ of the mean level spacing for earlier works, and more recently of the order of $10^{-4}$. By contrast, we are here concerned with the first few dozen eigenvalues, computed to very high accuracy, of the order of $10^{-12}$ of the mean level spacing or better.

The spectral staircase (number-counting) function for these systems is $N(E)=$ $\Sigma_{\mathrm{i}=1 \ldots \infty} \Theta\left(E-E_{i}\right)$, where $\Theta$ is the Heaviside unit step function. This is related to the spectral or trace function $\Phi(t)=\Sigma_{n=1 . . \infty} \exp \left(-E_{n} t\right)$ via a Laplace transform. Based on the work of several authors [18]-[21], with earlier work discussed extensively in Baltes 
and Hilf [22], the (smoothed) spectral staircase function (corresponding to the trace function appearing in [17]) for polygons is given by

$\mathrm{N}(\mathrm{E}) \sim[\mathrm{A} /(4 \pi)] \mathrm{E}+\left[\left(\mathrm{L}_{\mathrm{N}}-\mathrm{L}_{\mathrm{D}}\right) /(4 \pi)\right] \mathrm{E}^{(1 / 2)}+\left(\Sigma_{\mathrm{DD}, \mathrm{NN}}-\Sigma_{\mathrm{DN}, \mathrm{ND}}\right)\left[\left(\pi^{2}-\theta^{2}\right) /(24 \pi \theta)\right](1)$

where $A$ is the area, $L_{D}\left(L_{N}\right)$ is the length of that part of the perimeter having Dirichlet (Neumann) boundary condition, and the sums are over corner angles $\theta$ subtended by pairs of sides with boundary conditions as indicated. For basic unit length 2 for the two isospectral shapes of Figure 1 (which have the same area, perimeter lengths and corner angles), this reads, for the Nbc case, $\mathrm{N}_{\mathrm{N}}(\mathrm{E})=1.1141 \mathrm{E}+1.6302 \sqrt{\mathrm{E}}+0.4167$. In Figure 3, we plot $\mathrm{N}(\mathrm{E})$ for the first 31 modes for $\mathrm{Nbc}$ (including the zero mode). The agreement with this graph is good, demonstrating the need for inclusion of the zero mode and the plus sign for the second term in the case of Nbc compared with the minus sign for the Dbc case as was plotted in [6].

To our knowledge, no experiments involving isospectral shapes with Neumann boundary conditions have been performed, in contrast to reported experiments for the Dbc case [7], [8]. Some such Nbc experiments could be envisaged, however, based on acoustics and wave propagation in liquids [9, sect. 2.1], where Nbcs are involved. For instance, Blumel et al [23] reported on the nodal patterns of surface waves formed by agitating a tank with circular or stadium-shaped cylindrical walls. Chinnery et al. [24], [25] used a schlieren technique to visualize resonances in insonified water cavities with stadium and circular cross-sections. Hebert et al [26] made an experimental study of resonances of a fractal acoustic cavity.

It seems likely that these experimental techniques could be applied to crosssectional shapes as in Figure 1 to investigate their isopectrality. Independent checks on the accuracy of such experiments would be available through the sequential mode 
numbers, eigenvalue ratios, and nodal patterns of the analytical modes pictured in [17], along with the results in Table I and plots of Figure 2 in the present work.

\section{ALTERNATING BOUNDARY CONDITIONS}

There has been some work done on 2D systems with a mixture of Dirichlet and Neumann bcs on different parts of the boundary. Baltes and Hilf [22, p.47] show the appearance of a minus sign in the third (constant, corner-angle) term of the spectral number counting function for a rectangle whose sides successively alternate $\mathrm{Dbc}$ and $\mathrm{Nbc}$ (c.f. equation (1) above). In quantum billiards, there has been recent work where parts of a rectangular boundary have $\mathrm{Dbc}$ and parts have $\mathrm{Nbc}$, for raysplitting [13] and barrier [14] billiards. Thus it is important and timely to consider systems with both types of boundary conditions in detail.

Having verified, above, the accuracy of our modified domain-decomposition method for computationally handling Dirichlet or Neumann bcs in the case of the two standard provably isospectral shapes, we turn to the case of shapes with "alternating boundary conditions" (abcs) in which each side is successively Dbc or Nbc as one moves around the perimeter.

\section{A. Standard "bilby" and "hawk" shapes}

The isospectrality of the two standard shapes in the abc configuration has not been proved mathematically so far, and does not seem immediately amenable to the standard forms of proof. For instance, the transplantation method for unfolded 
domains described by Okada and Shudo [4] does not work here because a Dbc edge, upon folding, would yield a Dbc rather than an Nbc external edge as desired. Our aim here is to present strong numerical evidence for isopectrality in this new abc configuration. It can be seen that $\mathrm{A}, \mathrm{L}_{\mathrm{D}}, \mathrm{L}_{\mathrm{N}}$ and the $\theta$ s in equation (1) are the same for both shapes, so the coefficients of the three terms in (1) are equal for both, a necessary condition for isospectrality. In fact, there are two distinct such isospectral pair configurations. We denote by aDNbc (aNDbc) the situation for which the longest side in the alternating boundary condition configuration in each shape is chosen to have $\mathrm{Dbc}(\mathrm{Nbc})$.

Our numerical method now uses $\sin [(n+1 / 2) \alpha \theta]$ or $\cos [(n+1 / 2) \alpha \theta]$ in the Fourier-Bessel corner expansions, whichever conforms to the local bc. The computed eigenvalues for the first 30 modes of the two shapes (bilby and hawk) in the two abc configurations are given in Tables IIa and IIb. In either case, the results for both shapes in the same configuration agree to at least 12 significant figures. The nodal lines for the 10th modes for the two shapes in both configurations are shown in Figure 4. Unlike the cases of pure Dbc [5], [17] or pure Nbc [17], we have been unable to construct any exact "analytical" modes or to identify particularly simple nodal patterns in the computed eigenfunction plots. Thus independent checks as for the pure Dbc and Nbc cases do not seem to be available here.

The corresponding spectral staircase functions are plotted and compared with the graphs of equation (1) in Figure 5. For basic side length $h, A=(7 / 2) h^{2},\left|L_{N}-L_{D}\right|=$ $\sqrt{ } 2 \mathrm{~h}$, and the corner angle term has value $-5 / 12$. Thus, for $\mathrm{h}=2,(1)$ becomes $\mathrm{N}_{\mathrm{aDN}}(\mathrm{E})=$ 1.1141E $-0.2251 \sqrt{ } \mathrm{E}-0.4167$, with a plus sign for the second term in $\mathrm{N}_{\mathrm{aND}}(\mathrm{E})$. The 
plots show good agreement and confirm the minus sign for the third term in these cases of alternating boundary conditions for this pair of shapes.

\section{B. Other isospectral pairs}

Inspection of the building scheme as utilized by Even and Pieranski [8] for constructing isospectral pairs (with Dbc) from a basic building-block triangle shows that in general a 9-sided shape results; this would not support alternating boundary conditions. Their special case of an isosceles right-angled (90-45-45) triangle produces the standard, 8 -sided shapes. The question arises whether the bilby/hawk pair constitute the unique abc configuration isospectral pair, or whether there are other 8-sided shape pairs with the same property. In the notation of [8], it can be noted that the multiple-angle vertex $4 \gamma$ appears just once in the construction circuit, so if $\gamma=\pi / 4$ the number of sides is reduced by 1 from 9 to 8 . Such even-sided shapes are now amenable to alternating boundary conditions.

Further inspection of the shapes in Figure 1 of reference [8] shows that (in their notation) the multiple angles $3 \alpha$ and $3 \beta$ each appear twice, so they could not contribute to a change of parity of the number of sides. This may nevertheless suggest the possibility even of 6 -sided shapes. However, if, $\alpha($ or $\beta)=\pi / 3$, then for alternating sides the equality of the length differences $\mathrm{L}_{\mathrm{N}}-\mathrm{L}_{\mathrm{D}}$ for the pair of shapes as required by equation (1) leads to an inconsistency in the geometry of the fundamental buildingblock triangle.

Thus (in the notation of [8]) with $\gamma=45^{\circ}$, and neither of $\alpha$ and $\beta$ equal to $60^{\circ}$ or $45^{\circ}$, we get pairs of 8 -sided new shapes with, for a given pair, the same areas, the 
same corner angles, and the same $\left|\mathrm{L}_{N}-\mathrm{L}_{\mathrm{D}}\right|=\mathrm{a}+\mathrm{b}-\mathrm{c}>0$. We have chosen a non-isosceles building-block triangle with angles $\alpha=65^{\circ}, \beta=70^{\circ}, \gamma=45^{\circ}$ and side length $c=1$. These two new shapes are depicted in Figure 6. For either aDNbc or aNDbc pairs, the eigenvalues within a pair were computed using the method described above. Convergence was less rapid than for the bilby/hawk shapes, but we are confident in the agreement of the first 15 eigenvalues to at least 7 significant figures, as shown in Table III. Furthermore, even though these regions appear to be nearly mirror images, the nodal line patterns shown in Figure 7 for the 11th modes of the DN, and the ND, cases show significant topological differences. Altogether the numerical evidence of abc isospectrality beyond the standard bilby/hawk pair is compelling.

While the range of available examples is more restricted than in the pure Dirichlet or Neumann cases, it is remarkable that isospectrality persists with alternating boundary conditions for regions constructed according to rules that no longer provide a rigorous explanation of the phenomenon. 


\section{CONCLUSION}

The known isospectrality of the two standard shapes (Fig. 1) with Neumann boundary condition has been confirmed numerically to a high degree of accuracy, and good agreement with theory for the spectral staircase function was obtained.

We have presented numerical evidence that is the first and indeed strong indication of the isospectrality of these two standard shapes in the new boundary condition configurations with alternating Dirichlet and Neumann conditions on successive sides. A pair of non-standard isospectral shapes (Fig. 6) was similarly dealt with.

It is suggested that some experimental work involving fluids may illustrate the Nbc case, and that electromagnetic cavities might be relevant for the case of alternating boundary conditions.

\section{ACKNOWLEDGMENTS}

One of us (HPWG) would like to thank Professor Y. Okada for illuminating email correspondence. 
*Email address: driscoll@ math.udel.edu

**Email address: H.Gottlieb@griffith.edu.au

[1] M. Kac, Amer. Math. Monthly 73, 1 (1966).

[2] C. Gordon, D.L. Webb and S. Wolpert, Inventiones Math. 110, 1 (1992).

[3] S.J. Chapman, Am. Math. Monthly 102, 124 (1995).

[4] Y. Okada and A. Shudo, J. Phys. A 34, 5911 (2001).

[5] H. Wu, D.W.L. Sprung and J. Martorell, Phys. Rev. E 51, 703 (1995).

[6] T.A. Driscoll, SIAM Rev. 39, 1 (1997).

[7] S. Sridhar and A. Kudrolli, Phys. Rev. Lett. 72, 2175 (1994).

[8] C. Even and P. Pieranksi, Europhys. Lett. 47, 531 (1999).

[9] H.-J. Stockmann, Quantum Chaos - An Introduction (Cambridge University Press, Cambridge, 1999).

[10] Y. Hobiki, K. Yakubo and T. Nakayama, Phys. Rev. E 54, 1997 (1996).

[11] S. Russ, B. Sapoval and O. Haeberle, Phys. Rev. E 55, 1413 (1997).

[12] B. Gremaud and S.R. Jain, J. Phys. A 31, L637 (1998).

[13] A. Kohler and R. Blumel, Ann. Phys. 267, 249 (1998).

[14] J. Wiersig, Phys. Rev. E 65, 046217-1 (2002).

[15] H. Urakawa, Ann. scient. Ec. Norm. Sup. (4 ser) 15, 441 (1982).

[16] C. Gordon, D.L. Webb and S. Wolpert, Bull. Am. Math. Soc. 27, 134 (1992).

[17] H.P.W. Gottlieb and J.P. McManus, J. Sound Vibrat. 212, 253 (1998).

[18] B.D. Sleeman, IMA J. Appl. Math. 29, 113 (1982).

[19] H.P.W. Gottlieb, J. Austral. Math. Soc. Ser. B 26, 293 (1985). 
[20] H.P.W. Gottlieb, J. Austral. Math. Soc. Ser. B 29, 270 (1988).

[21] E.M.E. Zayed and A.I. Younis, Quart. Appl. Maths 48, 181 (1990).

[22] H.P. Baltes and E.R. Hilf, Spectra of Finite Systems (Bibliographisches Institut, Mannheim, 1976).

[23] R. Blumel, I.H. Davidson and W.P. Reinhardt, Phys. Rev. A 45, 2641 (1992).

[24] P.A. Chinnery and V.F. Humphrey, Phys. Rev. E 53, 272 (1996).

[25] P.A. Chinnery, V.F. Humphrey and C. Beckett, J. Acoust. Soc. Am. 101, 250 (1997).

[26] B. Hebert, B. Sapoval and S. Russ, J. Acoust. Soc. Am. 105, 1567 (1999). 
Table Captions

TABLE I. Eigenvalues of the first 30 non-zero modes, to 12 significant figures, for the two standard isospectral shapes of Fig. 1 (basic side 2 units), with Neumann boundary condition. (Mode number $\mathrm{M}=\mathrm{M}^{\prime}+1$.)

TABLE II. Eigenvalues of the first 30 modes, to 12 significant figures, for the two standard isospectral shapes of Fig. 1 (basic side 2 units), with (a) alternating Dirichlet (longest side) / Neumann boundary conditions; (b) alternating Neumann (longest side) / Dirichlet boundary conditions.

TABLE III. Eigenvalues of the first 15 modes, to 7 significant figures, for the two new non-standard isospectral shapes of Fig. 6, with (a) alternating Dirichlet (longest side) / Neumann boundary conditions; (b) alternating Neumann (longest side) / Dirichlet boundary conditions. 


\section{Figure Captions}

FIG. 1. The two standard isospectral shapes, termed respectively "bilby" and "hawk".

FIG. 2. Nodal lines for the 4th nontrivial Neumann bc modes.

FIG. 3. Spectral staircase for the bilby/hawk shapes with Neumann bc.

FIG. 4. Nodal lines for the $10^{\text {th }}$ modes in the aDNbc (top) and aNDbc configurations.

FIG. 5. Spectral staircases (vertical lines) and smooth approximations (dashed curves) for both types of alternating boundary conditions for the bilby/hawk pair.

FIG. 6. Two new isospectral shapes formed from a non-isosceles building-block triangle (with $\alpha=65^{\circ}, \beta=70^{\circ}, \gamma=45^{\circ}$ and $c=1$ in the notation of reference [8]).

FIG. 7. Nodal lines of the $11^{\text {th }}$ modes for the regions of Figure 6 , in the aDNbc (top) and aNDbc boundary configurations. While the two regions are nearly mirror images, their mode patterns in both these instances are quite different. 
TABLE I. Eigenvalues of the first 30 non-zero modes,

to 12 significant figures, for the two standard

isospectral shapes of Fig. 1 (basic side 2 units), with

Neumann boundary condition. (Mode number $\mathrm{M}=\mathrm{M}^{\prime}+1$.)

\begin{tabular}{llll}
\hline \hline $\mathrm{M}^{\prime}$ & $\mathrm{E}$ & $\mathrm{M}^{\prime}$ & $\mathrm{E}$ \\
\hline 1 & 0.211150088843 & 16 & 9.87841180931 \\
2 & 0.809165742343 & 17 & 10.3253541128 \\
3 & 1.05781354844 & 18 & 11.5754753532 \\
4 & 1.86039671198 & 19 & 11.6665580661 \\
5 & 2.46740110027 & 20 & 12.3370055014 \\
6 & 2.72788980382 & 21 & 12.9096372668 \\
7 & 3.39124050892 & 22 & 13.7742157078 \\
8 & 4.40632478595 & 23 & 15.4610825657 \\
9 & 4.93480220054 & 24 & 15.9519127351 \\
10 & 5.66570714255 & 25 & 16.2660218054 \\
11 & 5.81846754996 & 26 & 16.9514271289 \\
12 & 6.98274727028 & 27 & 19.0892095926 \\
13 & 7.64059043694 & 28 & 19.1962817413 \\
14 & 9.04934361203 & 29 & 19.7392088022 \\
15 & 9.86960440109 & 30 & 20.5304462804 \\
& & & \\
\hline
\end{tabular}


TABLE II. Eigenvalues of the first 30 modes, to 12 significant figures, for the two standard isospectral shapes of Fig. 1 (basic side 2 units), with (a) alternating Dirichlet (longest side) / Neumann boundary conditions;

(b) alternating Neumann (longest side) / Dirichlet boundary conditions.

\begin{tabular}{llll}
\hline \hline (a) & aDNbc & (b) & aNDbc \\
M & E & M & E \\
\hline 1 & 1.65141342709 & 1 & 0.715984505106 \\
2 & 1.90967315909 & 2 & 1.97361411622 \\
3 & 3.43870897236 & 3 & 2.77451509187 \\
4 & 4.17587478260 & 4 & 2.97462791606 \\
5 & 4.57209973453 & 5 & 3.88908349386 \\
6 & 5.62166889695 & 6 & 4.63290823432 \\
7 & 6.89161511324 & 7 & 6.02493079100 \\
8 & 7.74834106067 & 8 & 6.93817184169 \\
9 & 9.33008358225 & 9 & 7.77235813608 \\
10 & 9.80192274409 & 10 & 8.44522884854 \\
11 & 10.6260535933 & 11 & 9.39178897887 \\
12 & 11.5038125374 & 12 & 10.4460416956 \\
13 & 12.2664713847 & 13 & 10.9709402924 \\
14 & 12.8896196853 & 14 & 11.5756538487 \\
15 & 14.2405332523 & 15 & 12.8545214783 \\
16 & 15.5313799266 & 16 & 13.5117617705 \\
17 & 16.3655578572 & 17 & 15.0069728862 \\
18 & 17.3956907586 & 18 & 15.2660433735 \\
19 & 17.8925543463 & 19 & 15.9460361465 \\
20 & 19.2138859210 & 20 & 17.0144500638 \\
21 & 19.7290650856 & 21 & 17.8080832556 \\
22 & 20.3158331403 & 22 & 19.6769099441 \\
23 & 21.6332697571 & 23 & 20.2581964864 \\
24 & 22.2827868664 & 24 & 20.5724674001 \\
25 & 23.7501148857 & 25 & 21.8462979588 \\
26 & 24.2385971539 & 26 & 22.6047777014 \\
27 & 25.6971017156 & 27 & 23.5733311802 \\
28 & 26.2466652675 & 28 & 24.1261381785 \\
29 & 27.5323151203 & 29 & 24.6538070142 \\
30 & 28.0086952587 & 30 & 25.7410633370 \\
& & & \\
\hline & & & \\
1 &
\end{tabular}


TABLE III. Eigenvalues of the first 15 modes, to 7

significant figures, for the two new non-standard isospectral shapes of Fig. 6, with

(a) alternating Dirichlet (longest side) / Neumann boundary conditions;

(b) alternating Neumann (longest side) / Dirichlet boundary conditions.

\begin{tabular}{llll}
\hline \hline (a) & aDNbc & (b) & aNDbc \\
$\mathrm{M}$ & $\mathrm{E}$ & $\mathrm{M}$ & $\mathrm{E}$ \\
\hline 1 & 5.502422 & 1 & 2.267531 \\
2 & 6.449613 & 2 & 6.653412 \\
3 & 11.84158 & 3 & 9.217652 \\
4 & 12.62057 & 4 & 9.764155 \\
5 & 15.17411 & 5 & 12.18538 \\
6 & 18.20655 & 6 & 15.17442 \\
7 & 23.13378 & 7 & 19.74201 \\
8 & 26.76637 & 8 & 22.42403 \\
9 & 30.58768 & 9 & 27.25879 \\
10 & 33.11746 & 10 & 28.98984 \\
11 & 36.24867 & 11 & 31.97008 \\
12 & 37.13131 & 12 & 32.36155 \\
13 & 42.45487 & 13 & 36.06495 \\
14 & 44.50261 & 14 & 38.75119 \\
15 & 47.94645 & 15 & 40.87872 \\
& & & \\
\hline \hline
\end{tabular}



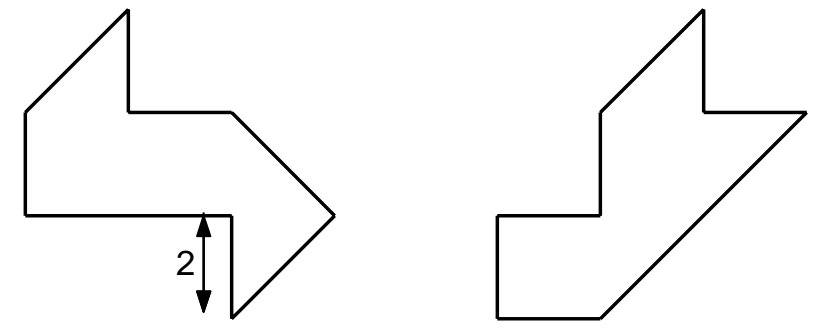

FIG. 1. The two standard isospectral shapes, termed respectively "bilby" and "hawk". 


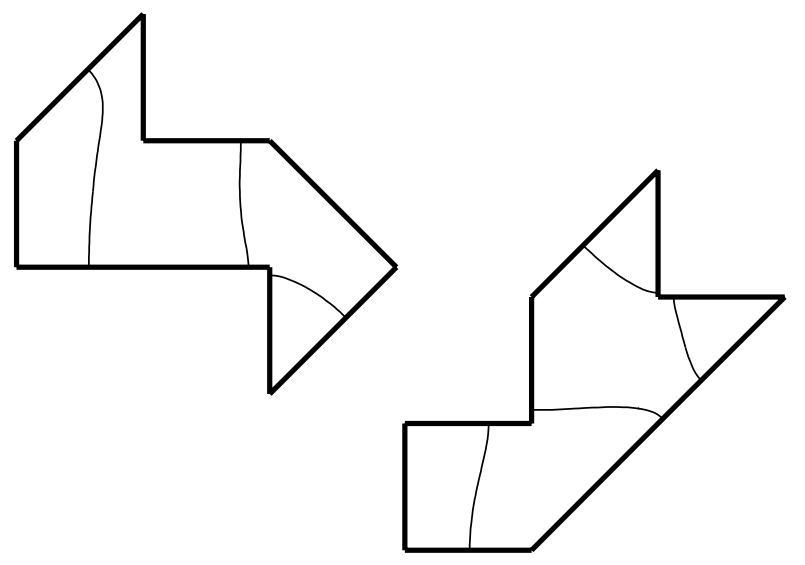

FIG. 2. Nodal lines for the 4th nontrivial

Neumann bc modes. 


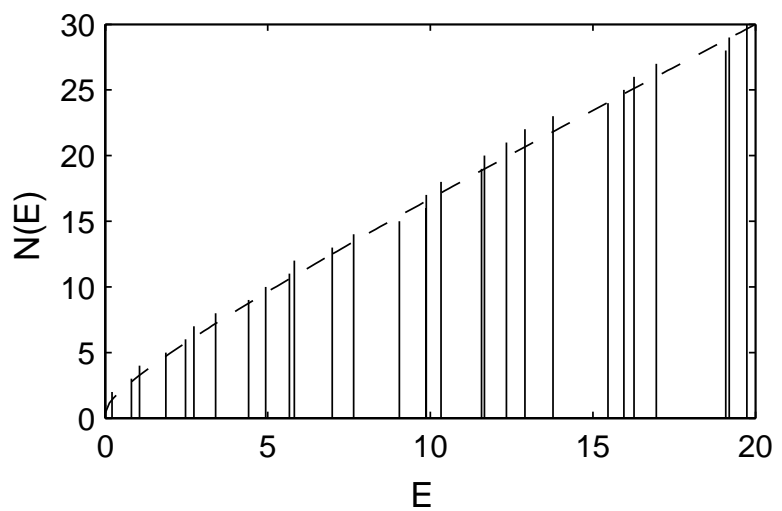

FIG. 3. Spectral staircase for the bilby/hawk shapes with Neumann bc. 

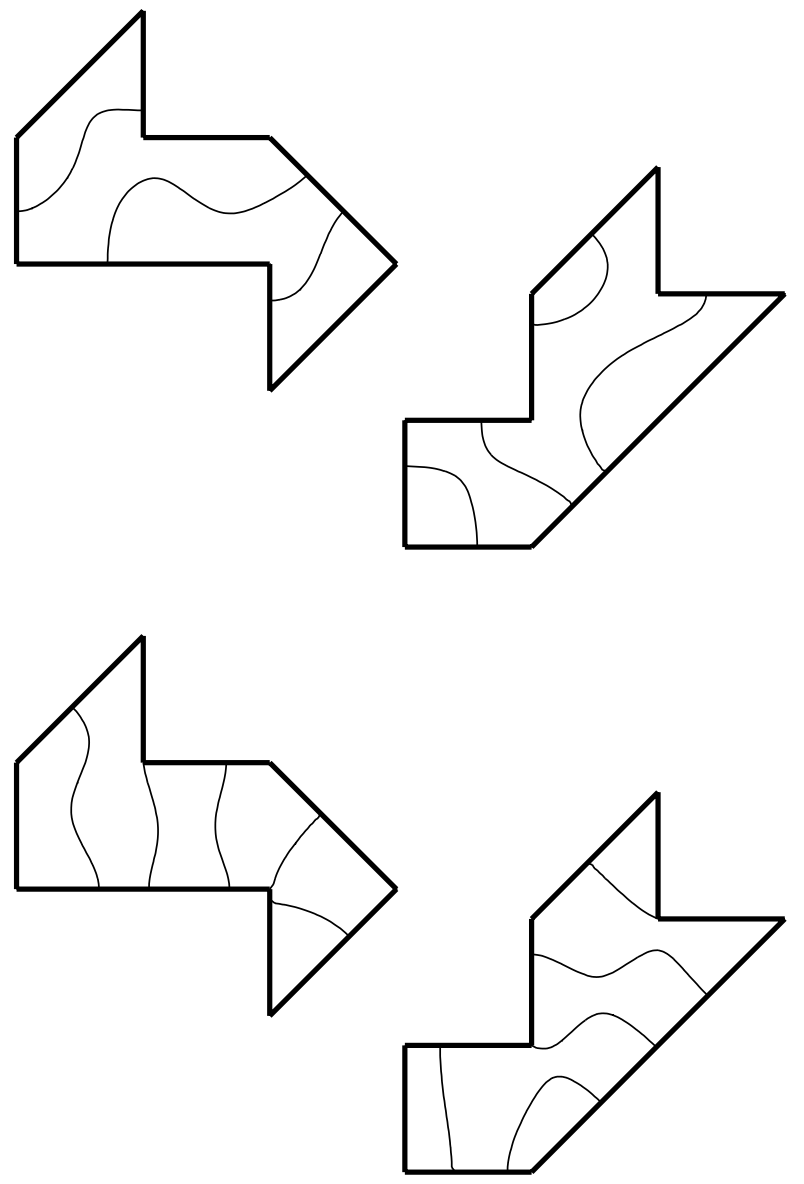

FIG. 4. Nodal lines for the $10^{\text {th }}$ modes in the aDNbc (top) and aNDbc configurations. 


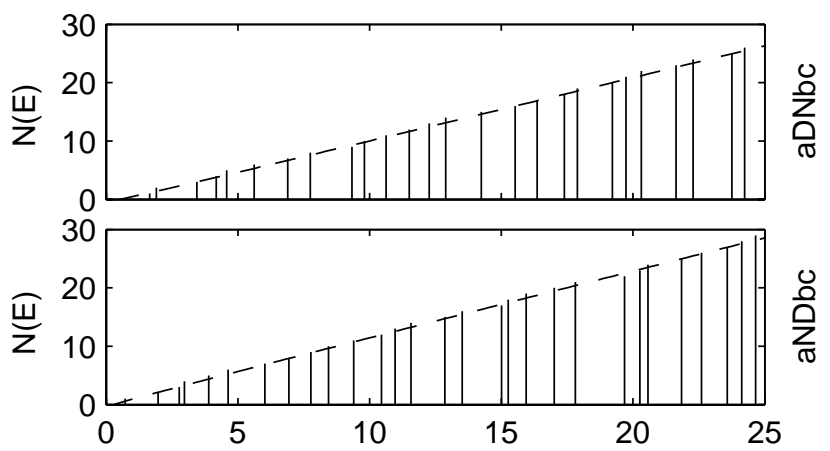

FIG. 5. Spectral staircases (vertical lines) and smooth approximations (dashed curves) for both types of alternating boundary conditions for the bilby/hawk pair. 

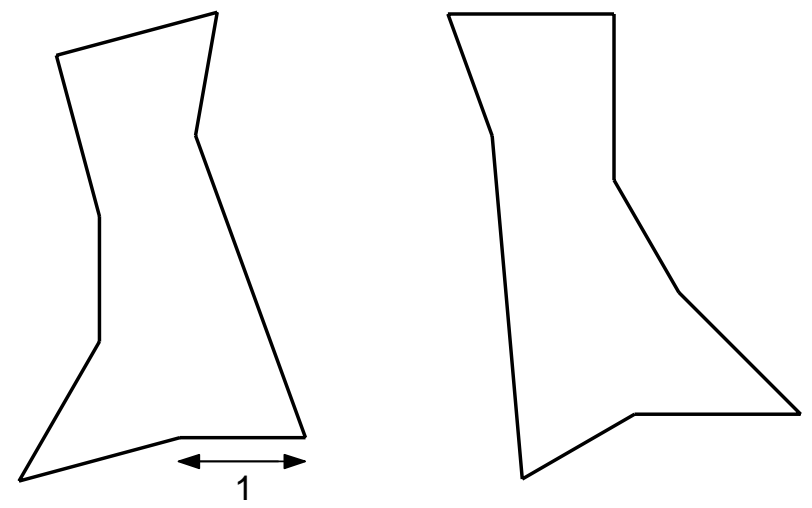

FIG. 6. Two new isospectral shapes formed

from a non-isosceles building-block triangle

(with $\alpha=65^{\circ}, \beta=70^{\circ}, \gamma=45^{\circ}$ and $c=1$ in the notation of reference [8]). 

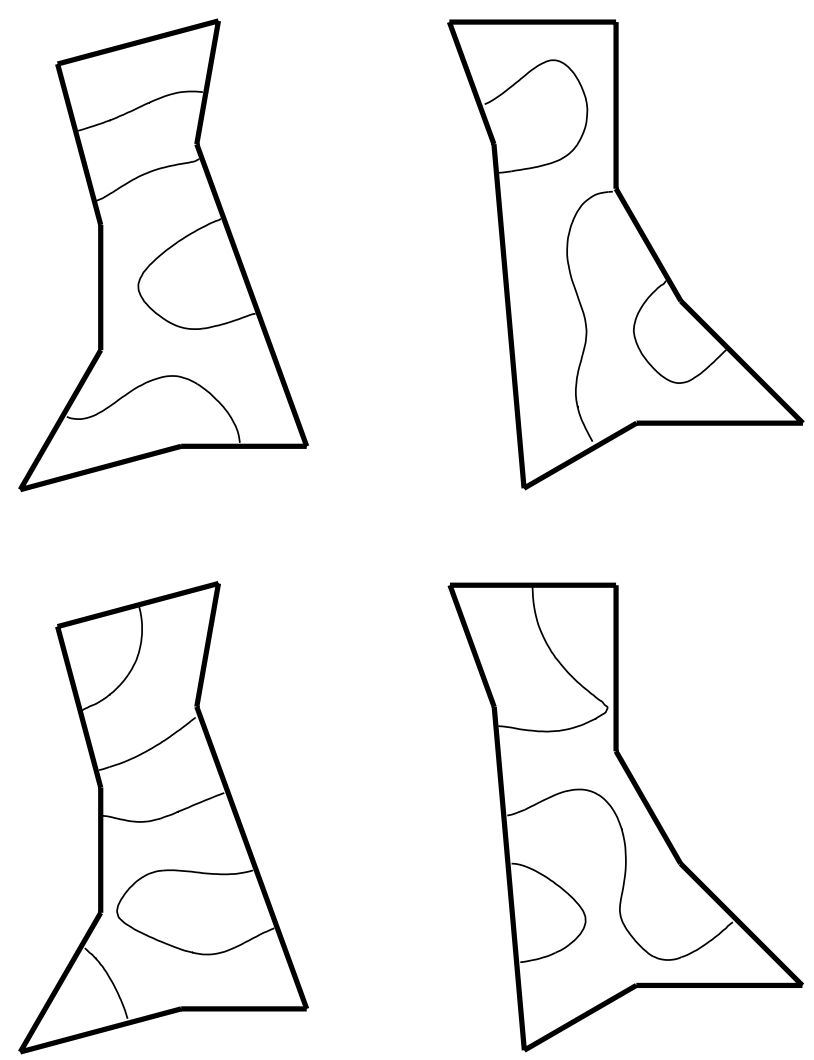

FIG. 7. Nodal lines of the $11^{\text {th }}$ modes for the regions of Figure 6, in the aDNbc (top) and aNDbc boundary configurations. While the two regions are nearly mirror images, their mode patterns in both these instances are quite different. 